\title{
Waiting time for cancer treatment and mental health among patients with newly diagnosed esophageal or gastric cancer: a nationwide cohort study
}

Huan Song ${ }^{1 *}$, Fang Fang ${ }^{1}$, Unnur Valdimarsdóttir ${ }^{1,2,3}$, Donghao Lu', Therese M.-L. Andersson ${ }^{1,4}$, Christina Hultman ${ }^{1}$, Weimin Ye ${ }^{1}$, Lars Lundell ${ }^{5}$, Jan Johansson ${ }^{6}$, Magnus Nilsson ${ }^{5}$ and Mats Lindblad ${ }^{5}$

\begin{abstract}
Background: Except for overall survival, whether or not waiting time for treatment could influences other domains of cancer patients' overall well-being is to a large extent unknown. Therefore, we performed this study to determine the effect of waiting time for cancer treatment on the mental health of patients with esophageal or gastric cancer.

Methods: Based on the Swedish National Quality Register for Esophageal and Gastric Cancers (NREV), we followed 7,080 patients diagnosed 2006-2012 from the time of treatment decision. Waiting time for treatment was defined as the interval between diagnosis and treatment decision, and was classified into quartiles. Mental disorders were identified by either clinical diagnosis through hospital visit or prescription of psychiatric medications. For patients without any mental disorder before treatment, the association between waiting time and subsequent onset of mental disorders was assessed by hazard ratios (HRs) with 95\% confidence interval (Cl), derived from multivariable-adjusted Cox model. For patients with a preexisting mental disorder, we compared the rate of psychiatric care by different waiting times, allowing for repeated events.

Results: Among 4,120 patients without any preexisting mental disorder, lower risk of new onset mental disorders was noted for patients with longer waiting times, i.e. 18-29 days (HR 0.86; 95\% Cl 0.74-1.00) and 30-60 days (HR 0.79; 95\% Cl 0.67-0.93) as compared with 9-17 days. Among 2,312 patients with preexisting mental disorders, longer waiting time was associated with more frequent psychiatric hospital care during the first year after treatment (37.5\% higher rate per quartile increase in waiting time; $p$ for trend $=0.0002$ ). However, no such association was observed beyond one year nor for the prescription of psychiatric medications.

Conclusions: These data suggest that waiting time to treatment for esophageal or gastric cancer may have different mental health consequences for patients depending on their past psychiatric vulnerabilities. Our study sheds further light on the complexity of waiting time management, and calls for a comprehensive strategy that takes into account different domains of patient well-being in addition to the overall survival.
\end{abstract}

Keywords: Mental disorder, Gastric cancer, Esophageal cancer, Cohort study, Cancer treatment, Waiting time

\footnotetext{
* Correspondence: huan.song@ki.se

1 Department of Medical Epidemiology and Biostatistics, Karolinska Institutet,

Box 281, Stockholm SE171 77, Sweden

Full list of author information is available at the end of the article
} 


\section{Background}

Given that the impact of tumor stage on survival is undisputed, it is problematic that the majority of tumors are diagnosed at a relatively advanced stage, when accelerated tumor growth and metastasis formation are imminent $[1,2]$. Therefore, it is logical to believe that a timely diagnostic work-up and treatment are of critical importance for the subsequent disease course and survival of the patients. However, previous studies, either from observational investigations $[3,4]$ or interventional studies ('Cancer waiting times targets' project [5], introduced by UK government in 2000 [6]) failed to show a clear survival benefit of shortened waiting time for cancer treatment. This was true even for relatively aggressive cancers, e.g. esophageal, gastric cancers $[7,8]$ and pancreatic cancer [6]. Yet, whether or not shorter waiting time for treatment influences other domains of cancer patients' overall well-being is to a large extent unknown.

Cancer patients have been shown to have increased risks of multiple mental disorders both immediately after cancer diagnosis and later on $[9,10]$. Knowledge about determinants for such risk increase may shed light on potential interventions. Due to the multimodality cancer diagnostics and therapies to date, waiting time for cancer treatment is likely to be increasing [11-13]. There is therefore an urgent need to clarify the impact of the extended waiting time on patient well-being in general, including the subsequent risk of mental disorders. To this end, we performed a cohort study to address the effect of waiting time for cancer treatment on the mental health of patients with newly diagnosed esophageal or gastric cancer.

\section{Methods}

\section{Database and study design}

Our study was based on the National Registry for Esophageal and Gastric Cancers (NREV), which included all patients with a diagnosis of esophageal or gastric cancer in Sweden. Details about this register, including its validity, have been described elsewhere [14]. Briefly, the register was officially launched in 2006, where patients were recruited from all health care providers diagnosing and managing gastric and esophageal cancers in Sweden. With regular checking with the Swedish Cancer Register to identify any potential additional patients, the NREV database has an average coverage of $92 \%$ for each calendar year [14]. Comprehensive information regarding diagnosis and treatment (mainly operations) was collected through questionnaires completed by the responsible physicians. The register was further cross-linked to the nationwide Cause of Death Register, Patient Register, Prescribed Drug register, and Emigration Register, obtaining information on follow-up outcomes of these patients.
In total, 7,984 patients with either an esophageal or gastric cancer were registered in NREV during 20062012. We excluded patients with conflict information (died or emigrated before diagnosis, $n=60$ ), or with missing (567) or incorrect (275) information on the date of treatment decision, leaving 7,080 patients $(88.7 \%)$ in the present analyses. All patients were followed until death, emigration out of Sweden, or December 31, 2012, whichever occurred first. This study was approved by the Regional Ethical Review Board in Stockholm, Sweden (Dnr 2013/596-31/3). Since we used de-identified register data, individual informed consent was not sought in line with institutional regulations.

\section{Waiting time for cancer treatment}

Since the exact starting date of cancer treatment was not available for patients without surgical treatment in NREV, we used the date of treatment decision as a proxy of treatment initiation for all participants. Waiting time for cancer treatment was accordingly defined as the time interval between cancer diagnosis and treatment decision. For most of the patients, treatment started several days after treatment decision. For patients that received primary surgical treatment (22\%), however, the actual waiting time until surgery might be postponed because of suboptimal physical status, routine pre-operation exams, or simply a queue to surgery. Nevertheless, a strong correlation was detected between the waiting time from cancer diagnosis to surgery and the waiting time between diagnosis and treatment decision (Pearson correlation coefficient $=0.86$ ).

\section{Ascertainment of mental disorders}

We ascertained mental disorders in two ways. Through cross-linkage to the Swedish Patient Register, we identified all inpatient or outpatient hospital visits with a mental disorder as one of the discharge or outpatient diagnoses, using the 10th Swedish revision of International Classification of Diseases codes (ICD-10: F10-F99). To complement the definition of mental disorders by using hospital diagnoses alone, we additionally assessed the use of psychiatric medications, by linking the cohort to Prescribed Drug Register. The selected psychiatric medications included antipsychotics (ATC code: N05A), anxiolytics (N05B) and antidepressants (N06A). Patients with any hospital visit or drug prescription with the above-mentioned ICD or ATC codes before treatment decision were defined as having a preexisting mental disorder. In sub-analyses, we specifically examined depression (ICD10: F32 or F33; ATC code N06A) and anxiety (ICD10: F40 or F41; ATC code N05B).

\section{Statistical analyses}

We first presented the basic characteristics of patients with different waiting times for treatment. The patients 
with a waiting time greater than 60 days $(n=648,9.2 \%)$ were then excluded from the following analyses, since such long delay was unusual and might allegedly reflect additional complexity of the disease or a strong wish of the patients. The remaining cancer patients were classified into four groups ( $\leq 8$ days, 9-17 days, 18-29 days, or 30-60 days), according to quartile distributions. Because a large proportion of patients presented with a history of mental disorder before treatment decision (35\%), we performed separately the primary analyses for patients with and without such history.

\section{Patients without previous mental disorders}

For 4,120 patients without any mental disorders before treatment decision, we followed them from date of treatment decision until first diagnosis of mental disorder (captured by either hospital visit or prescription of psychiatric drugs), emigration, death, or December 31, 2012, whichever occurred first. The association between waiting time and risk of mental disorder was examined by comparing the respective waiting time groups. We calculated hazard ratios (HRs) with their 95\% confidence intervals (CIs) by Cox model, adjusting for age at diagnosis, sex, marital status (single, married, divorce, or widow/widower), education level ( $<9$ years, 9-12 years, or $>12$ years), physical status (The American Society of Anesthesiologists (ASA) classification $<2$, or 2 and above), cancer type (esophageal or gastric cancer), pathological/ clinical (if pathological stage unavailable) stage (0-II stage, III stage, or IV stage), planned treatment type (curative, palliative, or supportive treatment), multidisciplinary (MDC) meeting (yes or no), admission pathway (general physician referral or emergency visit), and hospital volume (low, median, or high). Since a dramatically increased risk of mental disorders around cancer diagnosis has been reported [10], we used time since cancer diagnosis as the underlying timescale for Cox models after taking into account the delayed cohort entry (the number of days between cancer diagnosis and treatment decision). The proportional hazards assumption was checked graphically by Schoenfeld's partial residuals. Neither waiting time groups nor the above covariates appeared to violate this assumption. Cumulative incidence curves of mental disorders for different waiting time groups were graphed using the Nelson-Aalen method.

To assess potential effect modifiers of the studied associations, we performed sub-group analyses by different characteristics (e.g. sex, age group, etc.). We then separately analyzed mental disorders identified through hospital diagnosis alone and psychiatric medications alone assuming that mental disorders identified through hospital visits represent a severer phenotype compared to mental disorders identified through prescribed medications alone. Lastly, in addition to lumping together all mental disorders, we individually assessed HRs for depression and anxiety disorders.

\section{Patient with previous mental disorders}

For 2,179 patients with a mental disorder history before cancer diagnosis and 133 patients with a newly onset mental disorder during the waiting time for treatment, we compared the rate of psychiatric care (either through hospital visit or prescription of psychiatric medication) after treatment decision between different waiting time groups by extended Cox models (counting process model) [15], enabling inclusion of repeated events. Due to the overall violated proportional hazard assumption we divided the follow-up period ( $<1$ year and $\geq 1$ year) and fitted separate models. The Cox models were adjusted for all covariates stated above.

\section{Sensitivity analysis}

A sensitivity analysis was conducted among patients that received surgical treatment alone (without preoperative treatment, $n=1618$ ), for whom the waiting time to the actual treatment (surgery) could be estimated precisely. We repeated all the above-mentioned analyses to validate the results of the main analyses.

To assess potential impact of different time scales on the study results, we further used time since treatment decision, as well as time since 60 days after cancer diagnosis, as the underlying time scales in the Cox models.

A $p$ value less than 0.05 was considered to be statistically significant. All analyses were conducted in SAS statistical software, version 9.4 (Cary, NC).

\section{Results}

Table 1 illustrates the baseline characteristics of all participants, as well as by the different groups of waiting time for treatment. Overall, the mean age at the time of cancer diagnosis was 74 years and $65.7 \%$ of the patients were male. Patients with shorter waiting times generally had more advanced disease. In particular, patients with the shortest waiting time (1-8 days) tended to be older, with worse physical status, more advanced cancer stage and were more likely to be admitted through emergency visit. Most of these patients received their treatment decision without an MDC meeting (60.8\%). Since patients with the shortest waiting time obviously differed from the other patients, we used the second shortest waiting time group ('9-17 days') as the reference group in all following analyses.

\section{Patients without previous mental disorders}

Among patients without a previous history of mental disorder, 1,268 developed a mental disorder during followup. In general, we observed a decreased risk of mental disorder for patients with longer waiting times, compared 
Table 1 Characteristics of patients with a newly diagnosed esophageal or gastric cancer, by different waiting time groups

\begin{tabular}{|c|c|c|c|c|c|c|}
\hline \multirow[t]{2}{*}{ Characteristics } & \multirow[t]{2}{*}{ All } & \multicolumn{5}{|c|}{ Waiting time groups } \\
\hline & & $1-8$ days & 9-17 days & 18-29 days & $30-60$ days & $>60$ days \\
\hline Number of patients & 7080 & 1733 & 1542 & 1556 & 1601 & 648 \\
\hline \multicolumn{7}{|l|}{ Demographic factors } \\
\hline Age, mean $\pm S D$, years & $71.4 \pm 11.9$ & $73.7 \pm 12.5$ & $70.4 \pm 12.2$ & $70.4 \pm 11.6$ & $71.1 \pm 11.1$ & $70.3 \pm 11.1$ \\
\hline Gender ( $\%$ male) & 65.7 & 63.1 & 67.1 & 66.3 & 65.5 & 69.0 \\
\hline \multicolumn{7}{|l|}{ Material status, n (\%) } \\
\hline Single & $914(12.9)$ & 235 (13.6) & $227(14.7)$ & 180 (11.6) & 189 (11.8) & $83(12.8)$ \\
\hline Married & $3626(51.2)$ & 797 (46.0) & $806(52.3)$ & $843(54.2)$ & $842(52.6)$ & $338(52.2)$ \\
\hline Divorce & $1158(16.4)$ & $284(16.4)$ & $236(15.3)$ & $242(15.6)$ & $284(17.7)$ & $112(17.3)$ \\
\hline Widow/widower & $1368(19.3)$ & $411(23.7)$ & $271(17.6)$ & $288(18.5)$ & $283(17.7)$ & 115 (17.8) \\
\hline Missing & $14(0.2)$ & $6(0.3)$ & $2(0.1)$ & $3(0.2)$ & $3(0.2)$ & $0(0.0)$ \\
\hline \multicolumn{7}{|l|}{ Education level, n (\%) } \\
\hline$<=9$ years & $3070(43.4)$ & $778(44.9)$ & $655(42.5)$ & $643(41.3)$ & $700(43.7)$ & $294(45.4)$ \\
\hline $10-12$ years & $2561(36.2)$ & $586(33.8)$ & $545(35.3)$ & $578(37.2)$ & $600(37.5)$ & 252 (38.9) \\
\hline$>=12$ years & $1038(14.7)$ & $227(13.1)$ & $239(15.5)$ & $255(16.4)$ & $235(14.7)$ & $82(12.7)$ \\
\hline Missing & $411(5.8)$ & $142(8.2)$ & $103(6.7)$ & $80(5.1)$ & $66(4.1)$ & $20(3.1)$ \\
\hline \multicolumn{7}{|l|}{ Disease-related factors } \\
\hline \multicolumn{7}{|c|}{ Time of follow-up (months, until death, emigration or the end of study) } \\
\hline Mean \pm SD & $15.6 \pm 18.0$ & $10.4 \pm 16.1$ & $13.0 \pm 15.8$ & $16.3 \pm 17.7$ & $18.5 \pm 18.0$ & $26.6 \pm 21.5$ \\
\hline \multicolumn{7}{|l|}{ Hospital Volume } \\
\hline Low ( $<20$ cases/year) & $3310(46.8)$ & $975(56.3)$ & $749(48.6)$ & $664(42.7)$ & $659(41.2)$ & $263(40.5)$ \\
\hline Median (20-40 cases/year) & $1868(26.4)$ & $442(25.5)$ & $456(29.5)$ & $420(27.0)$ & $387(24.1)$ & $163(25.2)$ \\
\hline High ( $>40$ cases/year) & $1899(26.8)$ & $314(18.1)$ & $337(21.9)$ & $471(30.3)$ & $555(34.7)$ & $222(34.3)$ \\
\hline Missing & $2(0.0)$ & $0(0.0)$ & $1(0.0)$ & $0(0.0)$ & $0(0.0)$ & $3(0.0)$ \\
\hline \multicolumn{7}{|l|}{ ASA physical status, n (\%) } \\
\hline$|-| \mid$ & $4319(61.0)$ & $902(52.1)$ & $970(62.9)$ & $1023(65.8)$ & $1030(64.3)$ & $394(60.7)$ \\
\hline III-IV & $2588(36.6)$ & $791(45.6)$ & $529(34.3)$ & $494(31.8)$ & $539(33.7)$ & $235(36.3)$ \\
\hline Missing & $173(2.4)$ & $40(2.3)$ & $43(2.8)$ & $39(2.4)$ & $32(2.0)$ & $19(2.9)$ \\
\hline \multicolumn{7}{|l|}{ Cancer type, n (\%) } \\
\hline Gastric cancer & $3562(50.3)$ & $1004(57.9)$ & $788(51.1)$ & $737(47.4)$ & $715(44.7)$ & $318(49.1)$ \\
\hline Esophageal cancer & $3518(49.7)$ & $729(42.1)$ & $754(48.9)$ & $819(52.6)$ & $886(55.3)$ & $330(50.9)$ \\
\hline \multicolumn{7}{|l|}{ Cancer stage, n (\%) } \\
\hline Stage 0 & $191(2.70)$ & $24(1.38)$ & $24(1.56)$ & $29(1.86)$ & $52(3.25)$ & $62(9.57)$ \\
\hline Stage I & $905(12.8)$ & $131(7.56)$ & $157(10.2)$ & $226(14.5)$ & $260(16.2)$ & $131(20.2)$ \\
\hline Stage II & $924(13.1)$ & $155(8.94)$ & $200(13.0)$ & $252(16.2)$ & $236(14.7)$ & $81(12.5)$ \\
\hline Stage III & $2824(39.9)$ & $688(39.7)$ & $601(39.0)$ & $615(39.5)$ & $671(41.9)$ & 249 (38.4) \\
\hline Stage IV & $2188(30.9)$ & $726(41.9)$ & $557(36.1)$ & $423(27.2)$ & $369(23.1)$ & $113(17.4)$ \\
\hline Missing & $48(0.7)$ & $9(0.5)$ & $3(0.2)$ & $11(0.7)$ & $13(0.8)$ & $12(1.9)$ \\
\hline \multicolumn{7}{|l|}{ Admission type, n (\%) } \\
\hline GP referral & $5406(76.4)$ & $1071(61.8)$ & $1128(73.2)$ & $1303(83.7)$ & $1367(85.4)$ & $537(82.9)$ \\
\hline Emergency intake & $1517(21.4)$ & $627(36.2)$ & $367(23.8)$ & $225(14.5)$ & $201(12.6)$ & $97(15.0)$ \\
\hline Missing & $157(2.2)$ & $35(2.0)$ & $47(3.0)$ & $28(1.8)$ & $33(2.0)$ & $14(2.1)$ \\
\hline
\end{tabular}


Table 1 Characteristics of patients with a newly diagnosed esophageal or gastric cancer, by different waiting time groups (Continued)

\begin{tabular}{|c|c|c|c|c|c|c|}
\hline \multicolumn{7}{|c|}{ Treatment decided through multidisciplinary conference, n (\%) } \\
\hline Yes & $4080(57.6)$ & $668(38.6)$ & $929(60.3)$ & $990(63.6)$ & $1074(67.1)$ & $419(64.7)$ \\
\hline No & $2953(41.7)$ & $1053(60.8)$ & $599(38.9)$ & $556(35.7)$ & $521(32.5)$ & $224(34.6)$ \\
\hline Missing & $47(0.7)$ & $12(0.6)$ & $14(0.8)$ & $10(0.7)$ & $6(0.4)$ & $5(0.7)$ \\
\hline \multicolumn{7}{|c|}{ Planned treatment type, n (\%) } \\
\hline Curative & $3038(42.9)$ & $484(27.9)$ & $607(39.4)$ & $783(50.3)$ & $824(51.5)$ & $340(52.5)$ \\
\hline Palliative & $2631(37.2)$ & $714(41.2)$ & $663(43.0)$ & $547(35.2)$ & $548(34.2)$ & $159(24.5)$ \\
\hline Supportive & 1400 (19.8) & 531 (30.6) & $271(17.6)$ & $226(14.5)$ & $227(14.2)$ & $145(22.4)$ \\
\hline Missing & $11(0.1)$ & $4(0.3)$ & $1(0.0)$ & $0(0.0)$ & $2(0.1)$ & $4(0.6)$ \\
\hline
\end{tabular}

$S D$ standard deviation, ASA American Society of Anesthesiologists

to the reference group ('9-17 days') (Table 2); the multivariable adjusted HRs for all mental disorders were 1.07 (95\% CI $0.91-1.25)$ for a waiting time of ' $1-8$ days', 0.86 (95\% CI $0.73-1.01)$ for '18-29 days', and 0.79 (95\% CI $0.67-0.93$ ) for '30-60 days'. A clear trend of decreasing HRs with increasing waiting time was also noted ( $p$ for trend $<0.0001)$. Similar patterns of HRs were noted for mental disorders identified through hospital diagnosis or through drug prescription alone. The results for depression and anxiety did not differ largely from all mental disorders, although patients with the shortest waiting time appeared to have higher risk of anxiety, but not depression, compared to the reference group (Table 2).

Time since treatment decision (follow-up period), psychical status, cancer type, cancer stage, age group, education level, marital status, whether or not having MDC meeting, treatment plan or hospital volume did not modify these results further (Additional file 1: Table S1). The cumulative incidence curves (Fig. 1) illustrate the occurrence of mental disorders during follow-up by different groups of waiting times (since the curves didn't adjust for any covariates, they actually reflect a hypothetical scenario that all patients would survive during follow-up) and show that patients with longer waiting times had lower cumulative incidence rates of mental disorders after treatment decision.

\section{Patients with previous mental disorders}

Among 2179 patients with previous history of mental disorders, $68.8 \%$ received psychological care after the decision date of cancer treatment. Prolonged waiting time for cancer treatment was associated with an increased rate of hospital visits for mental disorders during the first year after treatment (Table 3), illustrating a 37.5\% higher rate of psychiatric hospital care per increasing waiting time $(95 \%$ CI 15.6-63.5\%, $p$ for trend $=0.0002$ ). This risk elevation was more pronounced for patients with only a history of psychiatric medication use but no hospital visit for mental disorders before treatment $(n=$ 1,361 ), for whom the rate of hospital visits increased by $49.0 \%$ per increasing waiting time group (95\% CI $18.4 \%-$ $87.4 \%)$ during the first year after treatment. The pattern was less clear beyond one year after treatment decision or for psychiatric medication use (Table 3).

\section{Sensitivity analysis}

Analyses restricted to patients that received surgical treatment alone yielded similar results as the primary analyses, with the exception that among patients with a history of psychiatric disorders $(n=473)$, the elevated rate of hospital visit for mental disorders by longer waiting times appeared also beyond one year after treatment

Table 2 Hazard ratios (HRs) and 95\% confidence intervals (Cls) ${ }^{*}$ for mental disorders among patients without mental disorder history $(n=4,120)$, by different waiting time groups

\begin{tabular}{llllll}
\hline Outcomes & Number of cases & $1-8$ days & $9-17$ days & $18-29$ days & $30-60$ days \\
\hline All mental disorders & 1268 & $1.07(0.91-1.25)$ & 1.00 (reference) & $0.86(0.73-1.01)$ & $0.79(0.67-0.93)$ \\
Mental disorders identified by in-/out-patient diagnosis & 129 & $0.79(0.47-1.34)$ & 1.00 (reference) & $0.85(0.54-1.33)$ & $0.56(0.34-0.93)$ \\
Mental disorders identified through drug prescription & 1227 & $1.09(0.93-1.29)$ & 1.00 (reference) & $0.88(0.75-1.04)$ & $0.82(0.69-0.97)$ \\
Depression (ICD10:F32,F33 or/and ATC N06A) & 317 & $0.96(0.69-1.34)$ & 1.00 (reference) & $0.91(0.67-1.23)$ & $0.66(0.48-0.92)$ \\
Anxiety (ICD10:F40,F41 or/and ATC N05B) & 799 & $1.21(0.99-1.48)$ & 1.00 (reference) & $0.87(0.71-1.06)$ & $0.84(0.68-1.03)$
\end{tabular}

*Adjusted for age, sex, marital status (single, married, divorce, widow/widower), education level (<9 years, 9-12 years, $>12$ years), physical status (The American Society of Anesthesiologists (ASA) classification <2, 2 and above), cancer type (esophageal/gastric cancer), stage (0, I, II, III, IV stage), and planned treatment type (curative, palliative, or supportive treatment), multidisciplinary meeting (yes/no), admission pathway (general physician referral / emergency intake), hospital volume (low, median, high) 


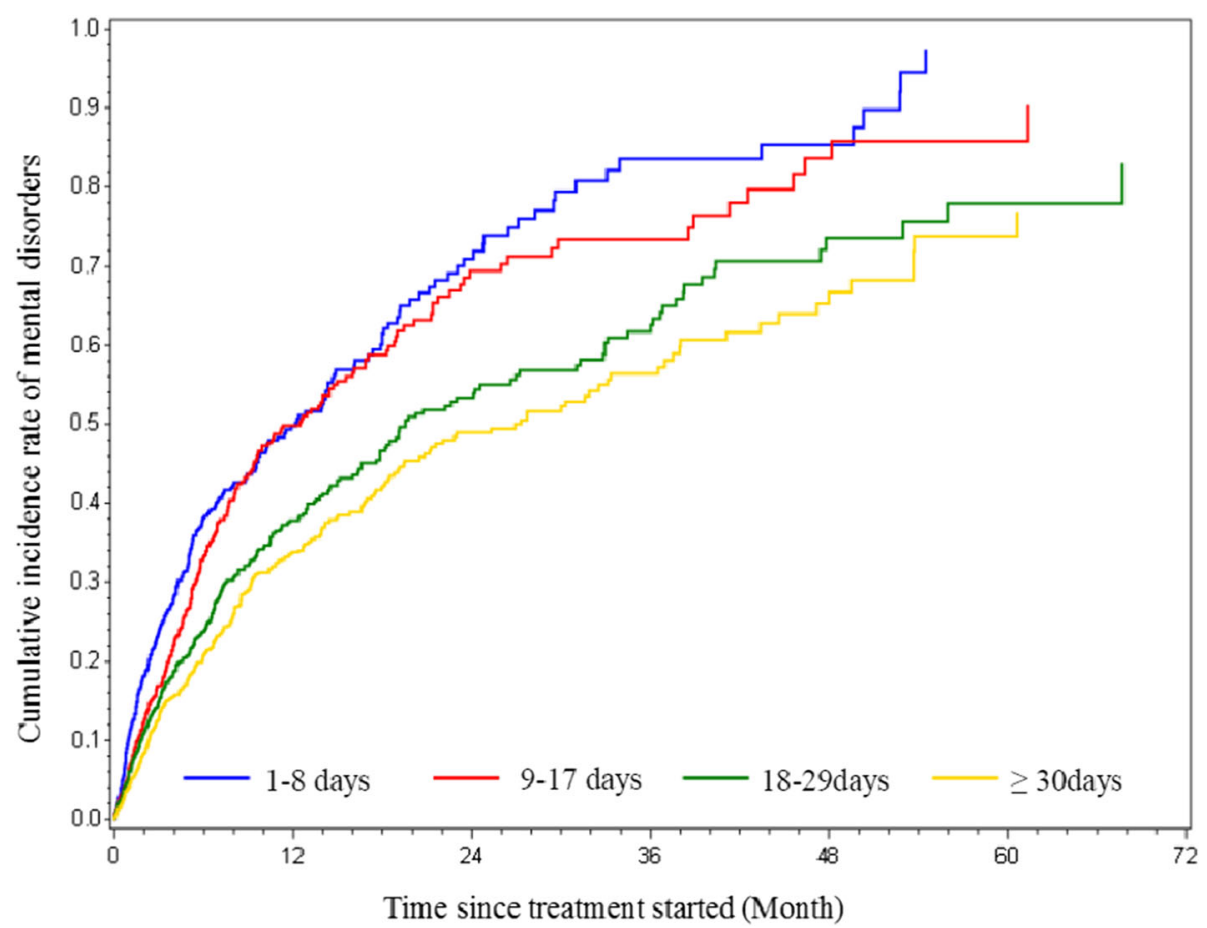

Fig. 1 Cumulative incidence rates of mental disorders among patients without preexisting mental disorders

(Additional file 2: Table S2). Alternative underlying time scales barely changed the results (data not shown).

\section{Discussion}

The impact of waiting time for treatment has mostly been studied in terms of cancer survival among patients with esophageal or gastric cancer $[8,16]$. To the best of our knowledge, this is the first large nationwide cohort study describing the association between waiting time for cancer treatment and mental health in patients with esophageal or gastric cancer. Our study sheds further light on the complexity of waiting time management, by highlighting a need to take into account different domains of patient well-being in addition to the overall survival. The complexity is further reflected by the potential differential impact of waiting time for treatment for different patients, depending on past psychiatric vulnerabilities. Specifically, while patients without any previous mental disorders may benefit from longer waiting times, in terms of future mental health, patients with previous mental disorders seem to benefit from a quick treatment decision.

Long waiting times for diagnosis and treatment are always of concern for the patients and the healthcare providers [5]. In Sweden, factors that could affect the waiting time of cancer treatment decision include the speed of the completion of staging information (mainly related to waiting time for radiological examinations), patients' wishes, as well as availability of MDC meeting (which normally hold once per week, meaning up to six

Table 3 Comparison of the rate of psychiatric care among patients with preexisting mental disorders $(n=2,312)$, by different waiting time groups

\begin{tabular}{|c|c|c|c|c|c|c|}
\hline \multirow{3}{*}{$\begin{array}{l}\text { Waiting } \\
\text { time } \\
\text { group }\end{array}$} & \multicolumn{6}{|c|}{ Hazard Ratios (HRs) and 95\% Confidence Intervals $(\mathrm{Cls})^{a}$} \\
\hline & \multicolumn{2}{|c|}{ All psychiatric cares } & \multicolumn{2}{|l|}{ Hopsital visits } & \multicolumn{2}{|c|}{ Medication prescriptions } \\
\hline & $\leq 1$ year & $>1$ year & $\leq 1$ year & $>1$ year & $\leq 1$ year & $>1$ year \\
\hline $1-8$ days & $0.90(0.74-1.10)$ & $0.68(0.44-1.07)$ & $0.80(0.49-1.31)$ & $0.36(0.14-0.91)$ & $0.90(0.74-1.10)$ & $0.72(0.46-1.13)$ \\
\hline 9-17 days & 1.00 (reference) & 1.00 (reference) & 1.00 (reference) & 1.00 (reference) & 1.00 (reference) & 1.00 (reference) \\
\hline 18-29 days & $1.04(0.86-1.26)$ & $0.84(0.51-1.24)$ & $1.49(0.98-2.25)$ & $0.80(0.38-1.72)$ & $1.01(0.83-1.23)$ & $0.79(0.51-1.22)$ \\
\hline 30-60 days & $1.09(0.91-1.32)$ & $0.70(0.47-1.02)$ & $2.01(1.24-3.24)$ & $0.51(0.24-1.07)$ & $1.03(0.85-1.24)$ & $0.71(0.48-1.04)$ \\
\hline
\end{tabular}

${ }^{a}$ Estimated by extended Cox model, adjusting for age, sex, marital status (single, married, divorce, widow/widower), education level (<9 years, $9-12$ years, $>12$ years), physical status (The American Society of Anesthesiologists (ASA) classification <2, 2 and above), cancer type (esophageal/gastric cancer), stage (0, I, II, III, IV stage), and planned treatment type (curative, palliative, or supportive treatment), multidisciplinary meeting (yes/no), admission pathway (general physician referral / emergency intake), hospital volume (low, median, high) 
days waiting time for some patients). According to our NREV database, $70 \%$ of the patients with esophageal or gastric cancer received treatment decision within 30 days after cancer diagnosis. The median waiting time for operation was however more than two months (67 days), and half of the patients received neoadjuvant therapy before the operation. Reduction in the medical delays before therapeutic intervention has proven to be of paramount importance for critical and urgent conditions such as stroke [17], myocardial infarction [18]. However, the importance of medical delays for chronic diseases including cancer is inconclusive. Most studies found null impact of waiting time on cancer specific survival, including gastroesophageal [7, 8, 16], colorectal [19], lung [20], and pancreatic [6] cancers. Other studies claimed however that a detrimental effect on the prognosis could be introduced if certain waiting time threshold was exceeded. For example, prolonged interval between diagnostic imaging and resection over 32 days for pancreatic cancer was associated with significantly increased risk of unexpected tumor progress as determined at laparotomy [21]. A recent population-based study [22] emphasized a significantly lower overall survival among patients with non-metastatic invasive breast cancer after 30 days waiting time for surgery (approximately 10\% decreasing survival for every 30 -day increment in waiting). Similarly, shorter overall survival has been suggested for 60 and more days of waiting time for head and neck cancers [23, 24], and for 12 and more days of waiting time for uterine cancers [25].

The psychological consequences of lengthy waiting time for treatment among cancer patients remain largely unknown. Our finding of the inverse assocation between waiting time and new onset mental disorders corroborates is unexpected, but corroborates the findings of a descriptive study including 21 breast cancer patients [26], which hypothesized that a proper interval between cancer diagnosis and start of treatment might be necessary and beneficial in terms of facilitating psychological adaptation [27]. A retrospective Dutch study including 202 breast cancer patients also implied that waiting time for diagnosis, but not waiting time for surgery, could affect the emotional well-being of cancer patients [28]. Considering that the pressure of providing speedy treatment might lead to reduced care quality $[29,30]$, short waiting time scheme should be designed and utilized with full caution.

Nevertheless, for patients with a history of mental disorders, the necessity of speedy cancer treatment seems to be justified. Clinical studies [31-33] indicated a similar cancer incidence but excess cancer mortality among patients with psychiatric diagnoses, compared to individuals without such comorbidity. Delayed diagnosis, and therefore a presentation at an advanced tumor stage at cancer diagnosis might be one possible explanation underlying such excess mortality [34]. However, UK researchers [35] observed a worse survival outcome for cancer patients with a history of mental illness, although these patients did indeed not have more advanced cancers at diagnosis, suggesting that the differential survival might instead be driven by factors during the process of cancer care after diagnosis. In the present study, we demonstrated that patients with history of psychiatric disorders with longer waiting times for cancer treatment had increased needs of hospital care for mental health during the first year after treatment. Psychiatric symptoms might affect patients' physical health, responsiveness to cancer treatment, and compliance to treatment regime (e.g. tolerance to intensive regimes) [33, 36], leading to altered overall survival in the long run. Improved prognosis might therefore be expected as a consequence of investment in timely treatment for such patients.

The major strength of our study is the large-scale population-based cohort design, with all information regarding cancer diagnosis, treatment, and mental disorders collected prospectively and independently. The linked data from death and emigration registers ensured the completeness of follow-up information. The availability of detailed questionnaire data from the quality register enabled considerations of a wide range of patient and tumor characteristics during the analysis.

Lack of data on the exact starting date of cancer treatment is a limitation. But the similar results of the sensitivity analysis by restricting to patients with surgical treatment alone relieved this concern. Further, the application of drug register for case identification, on one hand, increased the sensitivity by capturing undiagnosed patients who experienced difficulties in emotional responses [37], while reduced the possibility of underestimation caused by the prioritized cancer management-this might be especially true for patients in the shortest waiting group (i.e., more advanced diseases), being indicated by inconsistent HRs observed for diagnosed mental disorders and disorders identified by medication. However, on the other hand, the misclassification induced by this broad definition might exist, considering that antidepressant drug was not exclusively used for mental problems among cancer patients.

The potential influence of uneven mortality rates between patients with different waiting times (i.e., competing risks) on the present findings needs to be considered. However, informative censoring should have been alleviated sufficiently after adjusting for all prognostic indicators (i.e. cancer stage, therapy type, admission pattern, etc.) in the regression models. Also, similar results from subgroups analysis further relieved such concern. Moreover, although we cannot rule out possible residual confounding due to shared risk factors between cancer and mental disorders, the divergent result patterns between patients with and without mental disease history argues clearly such possibility. 


\section{Conclusions}

In conclusion, our study suggests the treatment delays after a diagnosis of esophageal or gastric cancer might be detrimental for the mental health of patients with preexisting mental disorder, at least during the first year after treatment. For patients without a mental disorder history, however, longer waiting times seem to be linked with a lower risk of new onset mental disorders after treatment.

\section{Additional files}

Additional file 1: Table S1. Subgroup analyses for patients with different characteristics. (DOC 74 kb)

Additional file 2: Table S2. Sensitivity analysis, restricted to patients with surgical treatments alone. (DOC $33 \mathrm{~kb}$ )

\section{Abbreviations}

ASA: The American Society of Anesthesiologists; Cl: Confidence interval; HR: Hazard ratio; ICD: International Classification of Diseases codes; MDC: Multidisciplinary; NREV: National Quality Register for Esophageal and Gastric Cancers

\section{Acknowledgments}

Not applicable.

\section{Funding}

This study was partly supported by the Swedish Cancer Society (grant No: CAN 2014/417).

\section{Availability of data and materials}

The data that support the findings of this study are available from a third party (i.e., Northern Regional Cancer Centre in Umeå as managers of NREV database, Swedish National Board of Health and Welfare, and Statistics Sweden) and but restrictions apply to the availability of these data, which were used under license for the current study, and so are not publicly available. Data are however available from the authors upon reasonable request and with permission of the third party.

\section{Authors' contributions}

Study concept and design: FF, UV, HS, MN, ML; data collection: JJ, LL; data analysis: HS, FF, TA, and DL; data interpretation: HS, FF, UV, TA, LL, JJ, MN, and $M L$; drafting of the manuscript: $H S, F F, U V, T A, D L, C H, W Y, L L, J J, M N$, and ML. All authors read and approved the final manuscript.

\section{Competing interests}

The authors declare that they have no competing interests.

\section{Consent for publication}

Not applicable.

\section{Ethics approval and consent to participate}

This study was approved by the Regional Ethical Review Board in Stockholm, Sweden (Dnr 2013/596-31/3). Since we used de-identified register data, individual informed consent was not sought in line with institutional regulations.

\section{Author details}

'Department of Medical Epidemiology and Biostatistics, Karolinska Institutet, Box 281, Stockholm SE171 77, Sweden. ${ }^{2}$ Center of Public Health Sciences, Faculty of Medicine, University of Iceland, Reykjavík, Iceland. ${ }^{3}$ Department of Epidemiology, Harvard T.H. Chan School of Public Health, Boston, MA, USA ${ }^{4}$ Department of Documentation \& Quality, Danish Cancer Society, Copenhagen, Denmark. ${ }^{5}$ Division of Surgery, Department of Clinical Science Intervention and Technology, Karolinska Institutet and Centre for Digestive
Diseases, Karolinska University Hospital, Stockholm, Sweden. ${ }^{6}$ Department of Surgery, Skåne University Hospital, Lund, Sweden.

Received: 5 August 2016 Accepted: 16 December 2016

Published online: 03 January 2017

\section{References}

1. Washington K. 7th edition of the AJCC cancer staging manual: stomach. Ann Surg Oncol. 2010;17(12):3077-9.

2. Rice TW, Blackstone EH, Rusch WW. 7th edition of the AJCC Cancer Staging Manual: esophagus and esophagogastric junction. Ann Surg Oncol. 2010;17(7):1721-4.

3. Matsuo K, Opper NR, Ciccone MA, Garcia J, Tierney KE, Baba T, Muderspach LI, Roman LD. Time interval between endometrial biopsy and surgical staging for type I endometrial cancer association between tumor characteristics and survival outcome. Obstet Gynecol. 2015;125(2):424-33.

4. Comber $\mathrm{H}$, Cronin DP, Deady S, Lorcain PO, Riordan P. Delays in treatment in the cancer services: impact on cancer stage and survival. Ir Med J. 2005;98(8):238-9.

5. Department of Health. The NHS cancer plan. Department of Health, London; 2000

6. Raptis DA, Fessas C, Belasyse-Smith P, Kurzawinski TR. Clinical presentation and waiting time targets do not affect prognosis in patients with pancreatic cancer. Surgeon. 2010;8(5):239-46.

7. Spahos T, Hindmarsh A, Cameron E, Tighe MR, Igali L, Pearson D, Rhodes M, Lewis MP. Endoscopy waiting times and impact of the two week wait scheme on diagnosis and outcome of upper gastrointestinal cancer. Postgrad Med J. 2005;81(961):728-30.

8. Sharpe D, Williams RN, Ubhi SS, Sutton CD, Bowrey DJ. The "two-week wait" referral pathway allows prompt treatment but does not improve outcome for patients with oesophago-gastric cancer. Eur J Surg Oncol. 2010;36(10):977-81.

9. Mitchell AJ, Chan M, Bhatti H, Halton M, Grassi L, Johansen C, Meader N. Prevalence of depression, anxiety, and adjustment disorder in oncological, haematological, and palliative-care settings: a meta-analysis of 94 interviewbased studies, Lancet Oncol. 2011:12(2):160-74.

10. Lu D, Andersson TM, Fall K, Hultman CM, Czene K, Valdimarsdottir U, Fang F. Clinical Diagnosis of Mental Disorders Immediately Before and After Cancer Diagnosis: A Nationwide Matched Cohort Study in Sweden. JAMA Oncol. 2016;2(9):1188-96

11. Stitzenberg KB, Sigurdson ER, Egleston BL, Starkey RB, Meropol NJ. Centralization of cancer surgery: implications for patient access to optimal care. J Clin Oncol. 2009:27(28):4671-8.

12. Bilimoria KY, Ko CY, Tomlinson JS, Stewart AK, Talamonti MS, Hynes DL, Winchester DP, Bentrem DJ. Wait times for cancer surgery in the United States: trends and predictors of delays. Ann Surg. 2011;253(4):779-85.

13. Liederbach E, Sisco M, Wang C, Pesce C, Sharpe S, Winchester DJ, Yao K Wait times for breast surgical operations, 2003-2011: a report from the National Cancer Data Base. Ann Surg Oncol. 2015;22(3):899-907.

14. Linder $G$, Lindblad M, Djerf P, Elbe P, Johansson J, Lundell L, Hedberg J. Validation of data quality in the Swedish National Register for Oesophageal and Gastric Cancer. Br J Surg. 2016;103(10):1326-35.

15. Guo Z, Gill TM, Allore HG. Modeling repeated time-to-event health conditions with discontinuous risk intervals. An example of a longitudinal study of functional disability among older persons. Methods Inf Med. 2008:47(2):107-16.

16. Visser E, Leeftink AG, van Rossum PS, Siesling S, van Hillegersberg R, Ruurda JP. Waiting Time from Diagnosis to Treatment has no Impact on Survival in Patients with Esophageal Cancer. Ann Surg Oncol. 2016;23(8):2679-89.

17. Fassbender K, Walter S, Liu Y, Muehlhauser F, Ragoschke A, Kuehl S, Mielke O. "Mobile stroke unit" for hyperacute stroke treatment. Stroke. 2003;34(6):e44.

18. Raghavan R, Benzaquen BS, Rudski L. Timing of bypass surgery in stable patients after acute myocardial infarction. Can J Cardiol. 2007;23(12):976-82.

19. Helewa RM, Turner D, Park J, Wirtzfeld D, Czaykowski P, Hochman D, Singh H, Shu E, Mckay A. Longer waiting times for patients undergoing colorectal cancer surgery are not associated with decreased survival. J Surg Oncol. 2013:108(6):378-84

20. Coughlin S, Plourde M, Guidolin K, Fortin D, Frechette E, Malthaner R, Inculet R. Is it safe to wait? The effect of surgical wait time on survival in patients with non-small cell lung cancer. Can J Surg. 2015;58(6):414-8.

21. Sanjeevi S, Ivanics T, Lundell L, Kartalis N, Andren-Sandberg A, Blomberg J, Del Chiaro M, Ansorge C. Impact of delay between imaging and 
treatment in patients with potentially curable pancreatic cancer. Br J Surg. 2016;103(3):267-75

22. Bleicher RJ, Ruth K, Sigurdson ER, Beck JR, Ross E, Wong YN, Patel SA, Boraas M, Chang El, Topham NS, et al. Time to surgery and breast cancer survival in the united states. JAMA Oncol. 2016;2(3):330-9.

23. van Harten MC, Hoebers FJ, Kross KW, van Werkhoven ED, van den Brekel MW, van Dijk BA. Determinants of treatment waiting times for head and neck cancer in the Netherlands and their relation to survival. Oral Oncol. 2015;51(3):272-8.

24. Murphy CT, Galloway TJ, Handorf EA, Egleston BL, Wang LS, Mehra R, Flieder DB, Ridge JA. Survival impact of increasing time to treatment initiation for patients with head and neck cancer in the United States. J Clin Oncol. 2016;34(2):169-78.

25. Elit LM, O'Leary EM, Pond GR, Seow HY. Impact of wait times on survival for women with uterine cancer. J Clin Oncol. 2014;32(1):27-33.

26. Drageset $S$, Lindstrom TC, Giske T, Underlid K. Being in suspense: women's experiences awaiting breast cancer surgery. J Adv Nurs. 2011;67(9):1941-51.

27. Lally RM. Acclimating to breast cancer a process of maintaining self-integrity in the pretreatment period. Cancer Nurs. 2010;33(4):268-79.

28. Henselmans I, Sanderman R, Smink A, Ranchor AV, de Vries J. Waiting times in breast disease clinics and psychological well-being: speedy care is better care. Ned Tijdschr Geneeskd. 2010;154:B491.

29. McConnell YJ, Inglis K, Porter GA. Timely access and quality of care in colorectal cancer: are they related? Int J Qual Health Care. 2010;22(3):219-28.

30. Porter G, Urquhart R, Kendell C, Bu J, McConnell Y, Grunfeld E. Timely access and quality of care in colorectal cancer: a population-based cohort study using administrative data. BMC Res Notes. 2013:6:355.

31. Guan NC, Termorshuizen F, Laan W, Smeets HM, Zainal NZ, Kahn RS, De Wit NJ, Boks MP. Cancer mortality in patients with psychiatric diagnoses: a higher hazard of cancer death does not lead to a higher cumulative risk of dying from cancer. Soc Psychiatry Psychiatr Epidemiol. 2013;48(8):128995.

32. Ishikawa H, Yasunaga H, Matsui H, Fushimi K, Kawakami N. Differences in cancer stage, treatment and in-hospital mortality between patients with and without schizophrenia: retrospective matched-pair cohort study. Br J Psychiatry. 2016;208(3):239-44.

33. Kisely S, Crowe E, Lawrence D. Cancer-related mortality in people with mental illness. JAMA Psychiat. 2013;70(2):209-17.

34. O'Rourke RW, Diggs BS, Spight DH, Robinson J, Elder KA, Andrus J, Thomas CR, Hunter JG, Jobe BA. Psychiatric illness delays diagnosis of esophageal cancer. Dis Esophagus. 2008;21(5):416-21.

35. Chang CK, Hayes RD, Broadbent MT, Hotopf M, Davies E, Møller H, Stewart R. A cohort study on mental disorders, stage of cancer at diagnosis and subsequent survival. BMJ Open. 2014;4(1):e004295.

36. He W, Fang F, Varnum C, Eriksson M, Hall P, Czene K. Predictors of discontinuation of adjuvant hormone therapy in patients with breast cancer. J Clin Oncol. 2015;33(20):2262-9.

37. Derogatis LR, Morrow GR, Fetting J, Penman D, Piasetsky S, Schmale AM, Henrichs M, Carnicke CLM. The prevalence of psychiatric-disorders among cancer-patients. JAMA. 1983;249(6):751-7.

\section{Submit your next manuscript to BioMed Central and we will help you at every step:}

- We accept pre-submission inquiries

- Our selector tool helps you to find the most relevant journal

- We provide round the clock customer support

- Convenient online submission

- Thorough peer review

- Inclusion in PubMed and all major indexing services

- Maximum visibility for your research

Submit your manuscript at www.biomedcentral.com/submit
Biomed Central 